\title{
LE BRUIT DE BARKHAUSEN DANS LES MATÉRIAUX FERROMAGNÉTIQUES
}

\author{
Par G. MONTALENTI, \\ Gruppo Nazionale Struttura della Materia del C.N.R., Istituto Elettrotecnico Nazionale Galileo Ferraris, Torino (Italia).
}

\begin{abstract}
Résumé. - Après avoir rappelé que l'effet de Barkhausen est dû aux discontinuités du processus d'aimantation, on décrit les faits expérimentaux connus lié au spectre de puissance du bruit de fond associé à ce processus.

Ce bruit peut être mesuré comme une tension aléatoire induite aux bornes d'une bobine entourant un noyau ferromagnétique dans lequel l'aimantation varie en fonction du temps. On observe que pour des fréquences de magnétisation entre $10^{-3}$ et $1 \mathrm{~Hz}$, le spectre de puissance est toujours compris entre quelques $\mathrm{Hz}$ et $10^{4} \mathrm{~Hz}$. La forme du spectre est presque indépendante du matériau, tandis qu'elle varie fortement si le circuit magnétique est ouvert ou fermé. L'intensité du bruit varie beaucoup pour des matériaux divers, tandis qu'elle reste pratiquement constante indépendamment $d u$ volume du matériau, si le noyau est massif. Pour un noyau laminé elle est proportionnelle au nombre d'anneaux.

Ces faits ne peuvent pas être interprétés par l'emploi des techniques mathématiques nécessaires pour expliquer le bruit de fond de courant d'un conducteur métallique massif. Il faut admettre que les impulsions de Barkhausen soient corrélées.

Si l'on emploie la théorie générale de Mazzetti pour le calcul du spectre de puissance d'événements corrélés, et si l'on admet que les impulsions élémentaires de Barkhausen ont la tendance à se grouper en avalanches, on peut très bien expliquer la plupart des faits observés.

Il reste encore à perfectionner cette théorie pour expliquer aussi la non-proportionnalité, particulièrement importante, de l'intensité du spectre à la fréquence de magnétisation $f_{\mathrm{m}}$ pour des valeurs de $f_{\mathrm{m}}$ supérieures à $0,1 \mathrm{~Hz}$. On doit enfin remarquer qu'aucune justification théorique n'a été donnée pour expliquer, même qualitativement, les importantes différences de bruit de Barkhausen de matériaux divers, et qu'il n'existe pas de critère pour établir un lien possible entre propriétés macroscopiques et bruit de Barkhausen.
\end{abstract}

Abstract. - The origin of Barkhausen noise is briefly recalled as being due to discontinuities of the magnetization process, and the experimental phenomena related to the power spectrum associated to this effect are described in detail.

The Barkhausen noise can be detected as a random voltage induced in a coil around a ferromagnetic core, whose magnetization is changing with time. For magnetization frequencies between $10^{-3}$ and $1 \mathrm{cps}$, the power spectrum is always found between some cps and $10^{4} \mathrm{cps}$. The shape of the spectrum is almost independent of the material, while it is widely different for closed or open magnetic circuits. The spectrum intensity varies depending on different materials, while it remains closely constant independently of the sample volume for bulk specimens. For laminated specimens it increases proportionally to the rings number.

These facts cannot be interpreted by means of the mathematical techniques necessary to explain the current noise of a bulk metallic conductor. One must admit that the Barkhausen pulses are correlated.

It is shown that by means of the general theory developed by Mazzetti to calculate the power spectrum of correlated events, and by supposing that the elementary Barkhausen pulses cluster into avalanches, one can very well account for most of the experimentally observed facts.

This theory must, as yet, be extended to account for the non proportionality of the power intensity to the magnetization frequency $f_{\mathrm{m}}$, which becomes particularly evident for $f_{\mathrm{m}}>0.1 \mathrm{cps}$. It is finally pointed out that no theoretical justification has been given so far to explain, even qualitatively, the important differences of the Barkhausen power intensity in different materials, and that no criterium exists to establish a possible relation between macroscopic properties and Barkhausen noise.

1. Introduction. - Il est bien connu qu'on doit à Barkhausen en 1919 la découverte que le processus d'aimantation d'un matériau ferromagnétique se produit par des sauts brusques. Ces discontinuités peuvent être observées comme des impulsions de tension électrique aux bornes d'un circuit d'induction entourant l'échantillon ferromagnétique soumis à un champ magnétique extérieur alternatif (fig. 1).

Cet effet doit être considéré comme la première preuve de l'existence de domaines de Weiss. Un matériau ferromagnétique est composé de plusieurs volumes élémentaires aimantés à saturation. Magnétiser
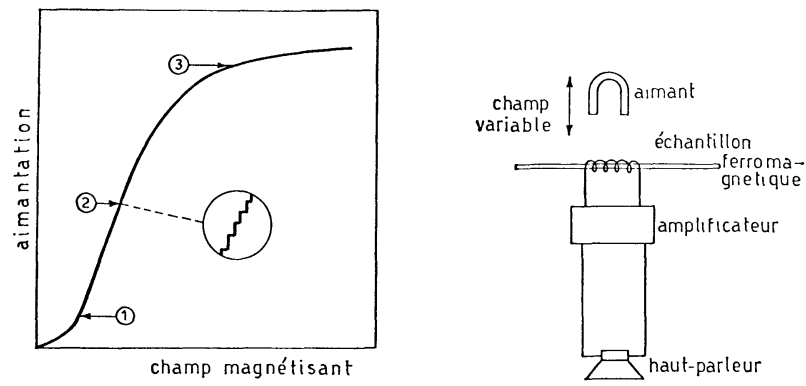

FIG. 1. - Expérience de Barkhausen. 
signifie faire tourner brusquement l'aimantation locale, pour l'orienter dans la direction du champ magnétique extérieur.

Depuis les expériences de Barkhausen, de nombreux travaux ont été publiés sur cet effet. Des auteurs ont cherché à déterminer le volume des domaines de Weiss; d'autres la forme des impulsions; d'autres encore la vitesse de propagation de ces impulsions. Une complète bibliographie peut être trouvée dans le récent volume de Stierstadt [1].

Cette conférence sera limitée à l'étude de l'effet de Barkhausen comme bruit de fond, qui se produit comme tension induite aux bornes d'un circuit entourant un matériau ferromagnétique soumis à un champ magnétique extérieur variable alternativement.

2. Résultats expérimentaux. - Il faut maintenant définir les modalités par lesquelles on mesure le spectre de puissance $\Phi(\omega)$, c'est-à-dire le carré de la tension efficace divisé par l'amplitude de bande du filtre. La tension totale de bruit est évidemment donnée par l'intégral de $\Phi(\omega)$ par respect à $\omega, \int_{-\infty}^{+\infty} \Phi(\omega) \mathrm{d} \omega$. La fréquence d'analyse est comprise entre $25 \mathrm{~Hz}$ et $20000 \mathrm{~Hz}$. La fréquence de magnétisation est inférieure à $1 \mathrm{~Hz}$ (entre $1 / 10 \mathrm{~Hz}$ et $1 / 1000 \mathrm{~Hz}$ ), de sorte que le spectre à raies dû à la non-ellipticité du cycle d'hystérésis ne soit pas inclus dans la mesure. Le schéma de principe de l'appareillage nécessaire à la mesure du spectre de puissance est donné en figure 2.

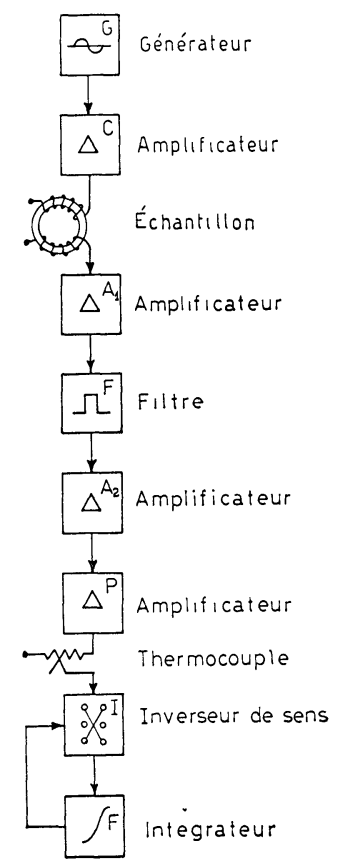

FIG. 2. - Schéma de l'appareillage employé pour la mesure du spectre de puissance du bruit de Barkhausen.

En figure 3, les spectres de différents matériaux sont donnés. On voit que l'amplitude de ces spectres est essentiellement nulle pour une fréquence d'analyse de l'ordre de $10^{4} \mathrm{~Hz}$ [2]. En figure 4, on peut voir que l'intensité du spectre est presque indépendante du volume de matériau ferromagnétique employé, pourvu qu'il soit massif [3]. En figure 5, on voit que l'intensité et la forme du spectre dépendent beaucoup du circuit magnétique : si le circuit est ouvert ou fermé, la forme du spectre est complètement différente [4].

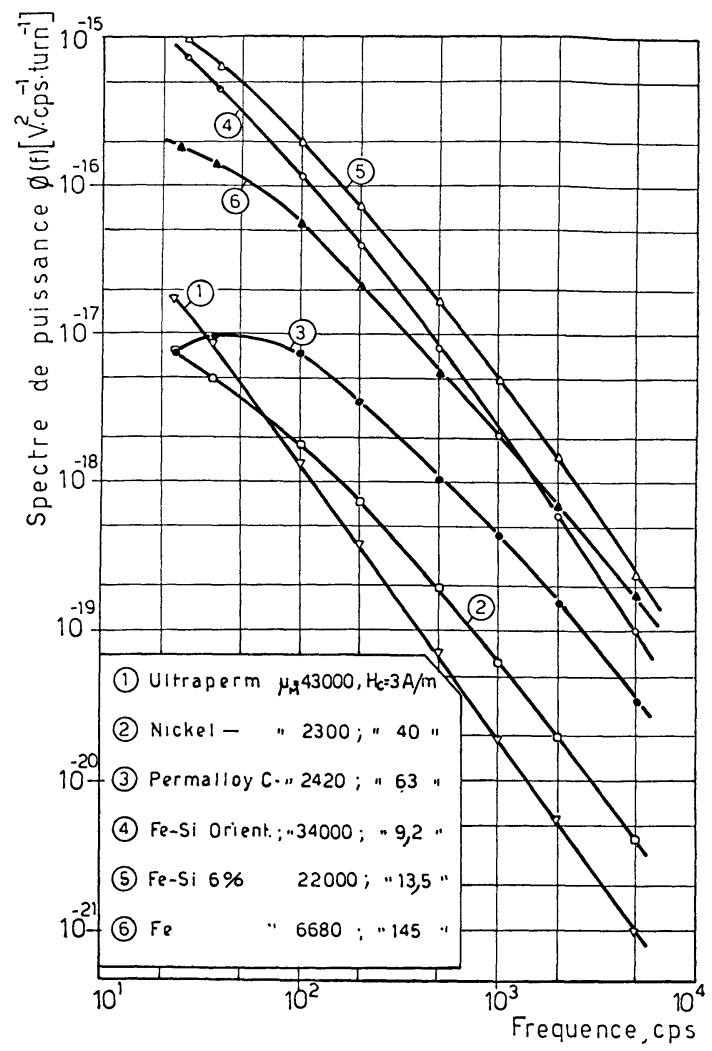

FIG. 3. - Spectre de puissance de différents matériaux en fonction de la fréquence d'analyse. Fréquence de magnétisation : $0,1 \mathrm{~Hz}$. Tous les spectres donnés à partir du volume d'échantillons divers et du nombre de spires de détection.

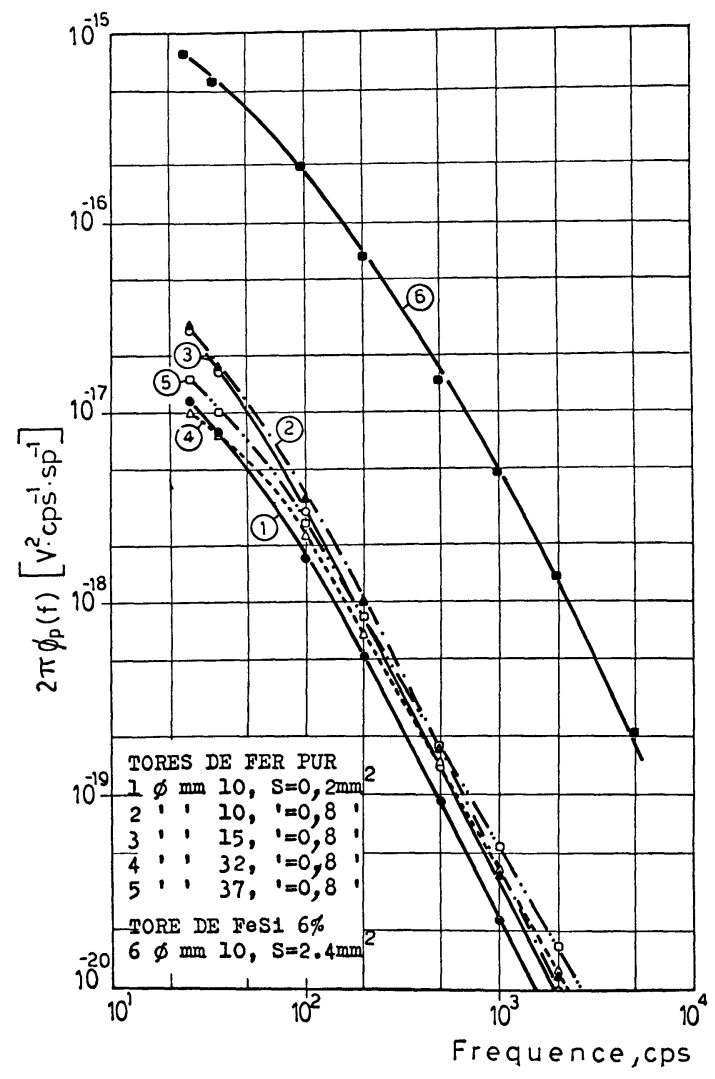

Fig. 4. - Spectres de puissance du bruit de Barkhausen mesurés sur des tores de fer pur ayant dimensions diverses, et sur un tore de $\mathrm{FeSi} 6 \%$. 


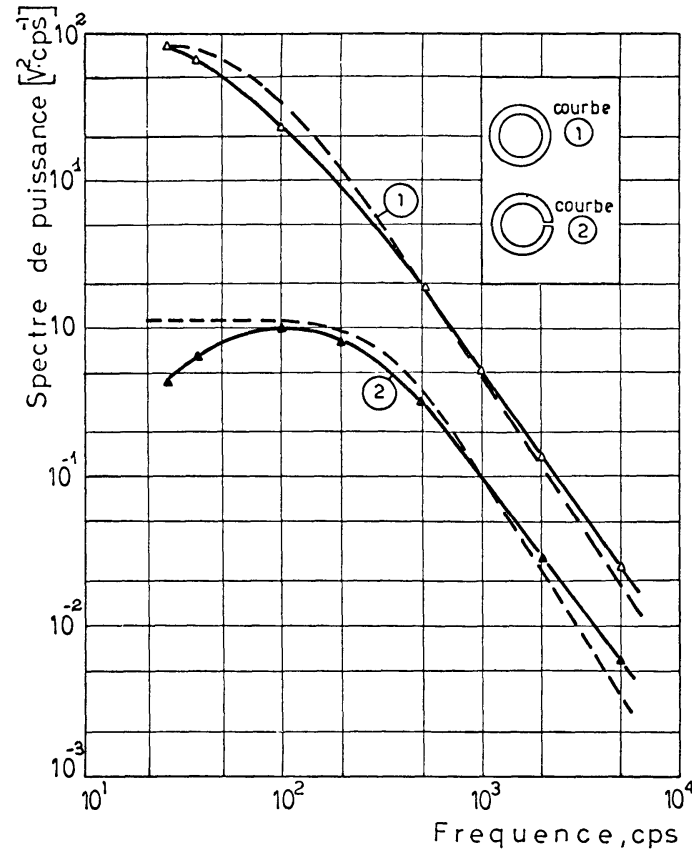

FIG. 5. - Spectres de puissance du bruit de Barkhausen mesurés sur un tore fermé (courbe 1) et sur un tore ouvert (courbe 2). Matériau : FeSi $6 \%$. Lignes continues : courbes expérimentales. Lignes hachées : courbes théoriques calculées par la (7).

De l'examen de tous ces résultats expérimentaux, on peut encore conclure que le spectre de puissance est en général une fonction décroissante de la fréquence, du type $f^{-n}$ où $n$ est proche de 2 , dans le champ des hautes fréquences d'analyse. Dans certains cas toutefois, $n$ peut atteindre une valeur maximum de 4 .

3. Interprétation des résultats expérimentaux. 3.1. Statistique de Sawada. - Tous les auteurs qui ont cherché à obtenir une expression théorique du spectre de puissance du bruit de Barkhausen $[5,6]$ ont employé les techniques mathématiques nécessaires pour expliquer le bruit de fond de courant.

On doit admettre, par hypothèse, que :

a) Toutes les impulsions sont égales et de forme exponentielle;

b) La probabilité de trouver une impulsion entre les temps $t$ et $(t+\mathrm{d} t)$ est indépendante de $t$.

Les événements sont donc considérés comme indépendants, c'est-à-dire non corrélés, et ils suivent par conséquent la statistique de Poisson :

$$
P(t) / n=\mathrm{e}^{-n t}
$$

où $n$ est le nombre moyen d'impulsions par unité de temps.

Un exemple de cette distribution est donné par les numéros de la roulette, qui ont tous la même probabilité d'être tirés. Toutefois la probabilité qu'un numéro donné soit tiré juste après un nombre $n$ de coups décroît comme indiqué par la (1).

Sous les hypothèses $a$ ) et $b$ ), on peut écrire, selon Campbell :

$$
\Phi(\omega)=n|S(\omega)|^{2}
$$

où $S(\omega)$ est la transformée de Fourier de l'impulsion élémentaire.
Si l'on fait l'hypothèse que ces impulsions sont des exponentiels, la (2) devient :

$$
\Phi(2 \pi f)=\frac{1}{2 \pi} \frac{f_{m} m a^{2}}{1 / \tau^{2}+(2 \pi f)^{2}}
$$

où $f_{m}$ est la fréquence de magnétisation, $m$ est le nombre moyen d'impulsions par cycle, $a$ est l'amplitude de l'impulsion, $\tau$ est la constante de temps de l'impulsion élémentaire, et $f$ la fréquence d'analyse. Suivant la (3), l'intensité du spectre de puissance devrait être proportionnelle au nombre d'impulsions par seconde, et donc à la fréquence de magnétisation. La forme du spectre donnée par la (3) est représentée en figure 6 .

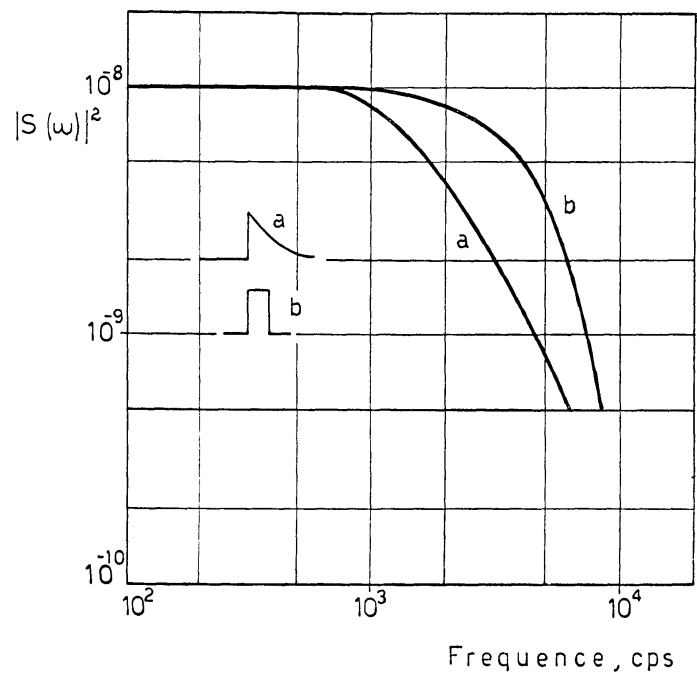

FIG. 6. - Densité spectrale d'une impulsion exponentielle (courbe a) et d'une impulsion rectangulaire (courbe b) ayant hauteur 1. Les courbes sont calculées sous l'hypothèse de l'indépendance statistique des impulsions.

Dans notre laboratoire, Biorci et Pescetti [7] ont montré qu'il y a une claire contradiction entre la (3) et les faits expérimentaux. En effet, les expériences sur des barreaux de fer ou de nickel ont permis d'observer que, aux basses fréquences d'analyse, l'intensité du spectre n'est pas proportionnelle à la fréquence de magnétisation, c'est-à-dire au nombre de sauts de Barkhausen par seconde. Ces résultats qui ont été confirmés par Warren [8, 9] et plus récemment encore dans notre laboratoire [4], sont résumés en figure 7. On voit que, aux basses fréquences d'analyse. l'intensité du spectre ne croît pas linéairement en fonction de la fréquence de magnétisation à partir de $10^{-2} \mathrm{~Hz}$.

Le problème d'interpréter ces résultats devient donc très difficile. Même si l'on laisse tomber l'hypothèse $a$ ) (égalité de toutes les impulsions), l'intensité du spectre de puissance reste toujours proportionnelle au nombre d'impulsions par seconde. Si l'hypothèse de l'indépendance des impulsions doit être conservée, même si l'on considère une distribution arbitraire de constantes de temps pour les impulsions élémentaires (comme il a été fait par Van der Ziel, par exemple, pour le bruit de flicker dans les semiconducteurs) [10], on ne réussit pas à expliquer les résultats montrés dans la figure. 


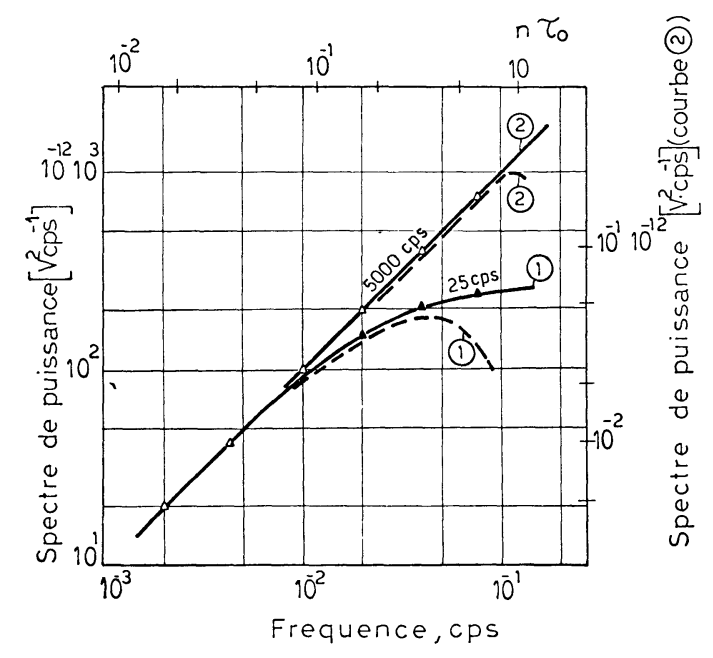

FIG. 7. - Densité de puissance du bruit de Barkhausen aux fréquences d'analyse de $25 \mathrm{~Hz}$ (courbe 1) et de $5000 \mathrm{~Hz}$ (courbe 2) en fonction de la fréquence de magnétisation. Matériau : FeSi $6 \%$. Lignes continues : courbes expérimentales. Lignes hachées : courbes théoriques basées sur la (7).

Il faut donc prendre la décision d'abandonner l'hypothèse que les impulsions soient indépendantes, c'est-à-dire qu'elles suivent la statistique de Poisson donnée par la (1).

Par la généralisation du théorème de Campbell, Mazzetti $[11,12]$ a donné une expression analytique très générale, qui permet d'obtenir le spectre de puissance pour des événements non indépendants. Il faut naturellement connaître la fonction de distribution et la forme de ces impulsions.

Il est donc maintenant question de décider quelle distribution doit être considérée au lieu de celle de Poisson. Sawada [13], et ensuite Bittel et Westerboer [14], ont obtenu expérimentalement, sur des barreaux de fer et de nickel, le résultat suivant : la fonction de distribution des impulsions de Barkhausen est bien représentée par la relation :

$$
P(t) / n=4 n t \mathrm{e}^{-2 n t}
$$

où $n$ est le nombre moyen d'impulsions par seconde et $t$ est le temps entre deux impulsions consécutives.

En figure 8, on représente les distributions de Poisson et de Sawada. On voit qu'il y a une grande différence entre les deux courbes. Si l'on tient compte de l'incrément du champ démagnétisant dû à une impulsion de Barkhausen, on peut donner une justification intuitive de la relation (4).

En supposant que toutes les impulsions ont la même amplitude et en employant la (4) dans les expressions générales données par Mazzetti $[11,12]$ pour le calcul du bruit de fond d'impulsions corrélées, on obtient : $\Phi(2 \pi f)$

$$
=\frac{1}{2 \pi}-\frac{m f_{\mathrm{m}} a^{2}}{1 / \tau^{2}+(2 \pi f)^{2}}\left(1-\frac{1}{2+(1 / 8)\left(2 \pi f / m f_{\mathrm{m}}\right)}\right)
$$

où $a$ est l'amplitude des impulsions, $m$ le nombre moyen d'impulsions par cycle, $f_{\mathrm{m}}$ la fréquence de magnétisation et $f$ la fréquence d'analyse.

La (5) prévoit une chute de l'intensité du spectre aux basses fréquences d'analyse de $50 \%$ au maximum, tandis que l'expérience montre qu'il y a des zones où l'intensité du spectre reste pratiquement constante pour fréquence de magnétisation croissante. D'ailleurs la constante de temps de l'impulsion élémentaire obtenue d'après les calculs et les expériences de Tebble [15] sur des fils très minces est de l'ordre de $10^{-4} \mathrm{~s}$, tandis qu'on obtient du spectre mesuré sur un tore des constantes de temps inférieures à $10^{-2} \mathrm{~s}$.

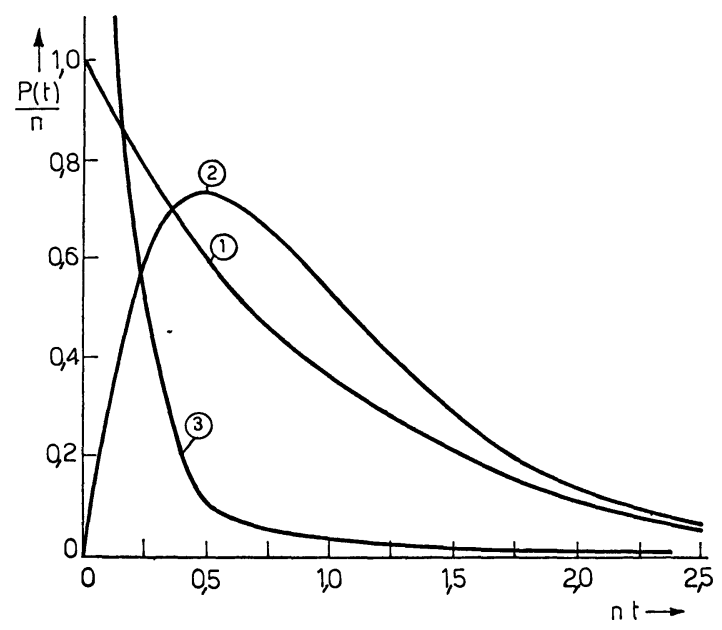

FIG. 8. - Fonctions de distribution selon statistiques diverses :

Courbe 1 : Statistique de Poisson (éq. (1)).

Courbe 2 : Statistique de Sawada (éq. (4)).

Courbe 3 : Statistique des avalanches (éq. (6)) (forme typique).

La variation de réluctance ne justifie pas cette réduction. Il faut encore remarquer que la (5) ne peut pas expliquer la considérable différence entre les spectres mesurés sur des tores ouverts ou fermés (voir fig. 5) que nous avons déjà remarquée.

Mais la plus grande discordance peut être mise en évidence si l'on calcule de la (5) l'intensité du spectre aux basses fréquences d'analyse, et on la compare aux valeurs expérimentales. La valeur calculée est à peu près mille fois plus petite que celle observée. Pour ce calcul, on a considéré une valeur de $10^{-4} \mathrm{~s}$ pour la constante de temps $\tau$, tandis que le nombre d'impulsions par cycle $m$ et l'amplitude de l'impulsion $a$ ont été choisis de sorte que, aux hautes fréquences d'analyse, les valeurs du spectre calculées soient coïncidentes avec celles observées.

Cette contradiction et cette impossibilité d'interpréter certains faits de particulière importance demandent que l'examen des faits expérimentaux soit amélioré.

3.2. Statistique Des GRANDES impulsions DE Barkhausen. - Les résultats montrent qu'une amélioration de l'interprétation peut seulement être faite par la substitution de la statistique de Sawada [13] donnée par la (4) avec une statistique plus proche des faits expérimentaux. Déjà Bozorth [16], par des observations à l'oscilloscope, et plus tard Bittel et Westerboer [14], par des méthodes de compte, avaient signalé que les impulsions élémentaires de Barkhausen peuvent se grouper et donner lieu à de grandes discontinuités de Barkhausen, comme montré en figure 9. Il est particulièrement important de souligner que ces grandes discontinuités, comme l'on voit sur la figure, sont formées par une avalanche, ou mieux par un groupement d'impulsions élémentaires, et ne sont pas 


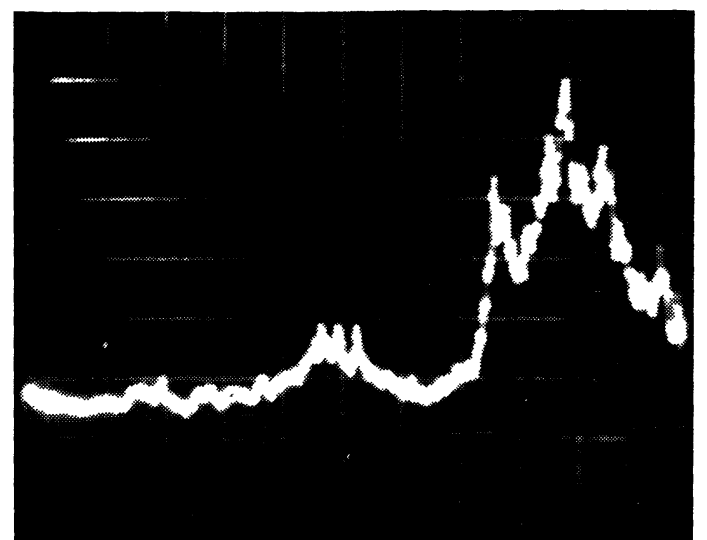

FIG. 9. - Observation à l'oscilloscope d'une grande discontinuité de Barkhausen formée par une avalanche de plusieurs sauts plus petits, mesurée sur un tore de fer. (Ordonnées : tension $=100 \mu \mathrm{V} / \mathrm{div}$; abscisses : temps $=0,01$ s'div.)

dues à l'inversion brusque de domaines de Weiss de grandes dimensions. Il faut donc trouver une nouvelle fonction de distribution pour la substituer aux statistiques de Poisson (éq. (1)) et de Sawada (éq. (4)). Cette fonction doit tenir compte de la tendance des impulsions élémentaires à se grouper. Si $\tau$ représente la durée de l'impulsion élémentaire, la probabilité que d'autres impulsions tombent dans un délai $\tau^{\prime}<\tau$ doit être beaucoup augmentée.

On peut donc admettre comme fonction de distribution la suivante :

$$
P(t)=K_{1} \mathrm{e}^{-\mu_{1} t}+K_{2} \mathrm{e}^{-\mu_{2} t} .
$$

Cette fonction est représentée par la courbe 3 en figure 8 . On voit que la première partie de la courbe est caractérisée par des ordonnées bien plus grandes que celles de la courbe de Poisson : la probabilité que les impulsions se suivent très proches a donc augmenté. La deuxième partie représente au contraire la courbe normale de Poisson. La substitution de la (6) dans l'expression générale donnée par Mazzetti [12] pour le calcul du spectre de puissance conduit à la formule suivante :

$\Phi(2 \pi f)$

$=\varphi(2 \pi f)\left[1+\frac{2 \rho\left(1-n \tau_{0}\right)^{2}}{(2 \pi f)^{2} \tau_{0}^{2} \rho^{2}\left(1-n \tau_{0}\right)^{2}+1}\right]$

où $\rho$ est le nombre d'impulsions par groupe; $\tau_{0}$ est l'intervalle de temps moyen entre deux impulsions consécutives dans le groupe; $n$ est le nombre moyen d'impulsions par seconde; $\varphi(2 \pi f)$ est le spectre de puissance d'impulsions non corrélées, dont l'expression est donnée par la (3).

Il faut observer que la (7) dépend très peu de la forme de la relation (6), et dépend surtout des valeurs de $\rho, \tau_{0}, n$ qu'on a définis tout à l'heure. On doit donc penser que cette fonction représente, dans la forme la plus générale, le spectre de puissance pour des impulsions qui se groupent en avalanches. Si au contraire il s'agissait de grandes impulsions isolées, non corrélées, la statistique de Poisson serait toujours valable, et on ne pourrait pas expliquer la non-proportionnalité de l'intensité du spectre au nombre d'impulsions par seconde, c'est-à-dire la non-proportionnalité à la fréquence de magnétisation.
La (7) doit maintenant être vérifiée expérimentalement :

a) Premièrement, on supposera que la fréquence de magnétisation soit suffisamment basse de sorte que $n \tau_{0}$ (c'est-à-dire le produit du nombre moyen d'impulsions par seconde par la valeur moyenne de l'intervalle de temps entre deux impulsions dans le groupe) soit beaucoup plus petit que l'unité. Le facteur $\varphi(2 \pi f)$ est le spectre de puissance qu'on devrait observer si les impulsions élémentaires étaient statistiquement indépendantes, au lieu d'être corrélées en groupes, et il est donc constant (puisque la constante de temps de l'impulsion élémentaire est à peu près de $10^{-4} \mathrm{~s}$ ) jusqu'à des fréquences d'analyse de l'ordre de $10^{4} \mathrm{~Hz}$. Si la valeur de $\rho \tau_{0}$ (c'est-à-dire de la durée moyenne du groupe d'impulsions qui forment une grande discontinuité de Barkhausen) est considérée de $2,5 \times 10^{-3} \mathrm{~s}$, on obtient un très bon accord entre la courbe expérimentale et la courbe calculée ( fig. 10). On remarque que la valeur choisie pour $\rho \tau_{0}$ a été estimée par des observations à l'oscilloscope sur la durée de l'avalanche.

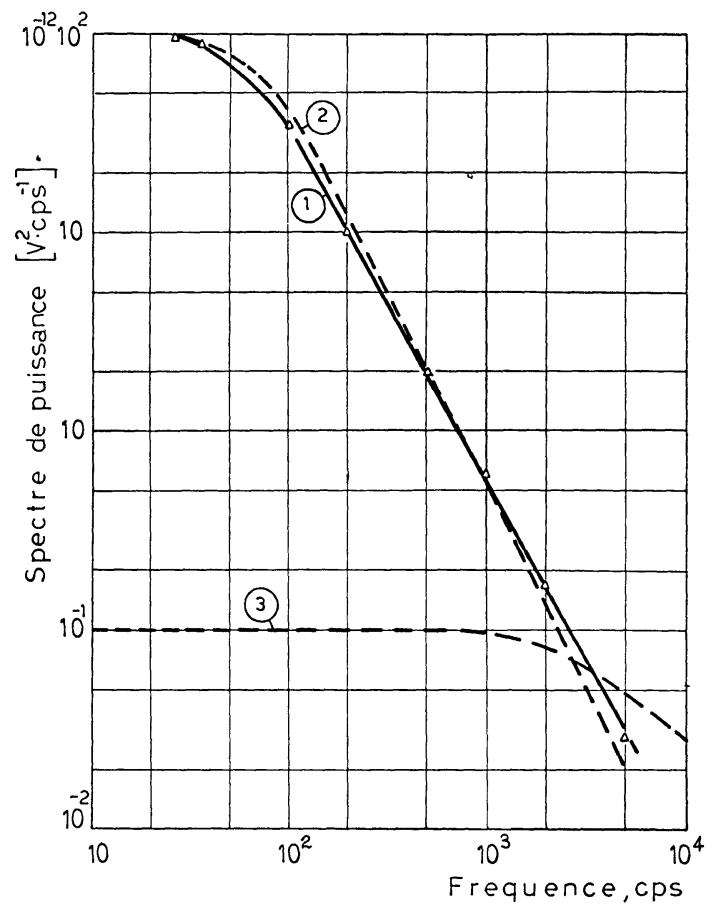

Fig. 10. - Courbe 1 : Spectre de puissance du bruit de Barkhausen sur tore de FeSi $6 \%$. Fréquence de magnétisation : $0,01 \mathrm{~Hz}$.

Courbe 2 : Spectre de puissance théorique donné par la (7) tenant compte du groupement des impulsions $\left(\rho \tau_{0}=2,5 \times 10^{-3} \mathrm{~s}\right)$.

Courbe 3 : Spectre de puissance théorique calculé sous hypothèse d'indépendance statistique des impulsions de Barkhausen.

Il est d'ailleurs clair que la courbe théorique est connue avec la seule indétermination d'une constante de proportionnalité. Cette constante est obtenue de mesures aux hautes fréquences d'analyse. Toutefois, on insiste sur le fait que si les impulsions étaient élémentaires et non corrélées l'intensité du spectre aurait été mille fois plus petite que celle expérimentale. Le groupement multiplie donc par $\rho$ (nombre d'impulsions par groupe) l'intensité du spectre aux basses 
fréquences d'analyse, et réduit la fréquence de coupure du spectre par le rapport entre la durée du groupe et la constante de temps de l'impulsion élémentaire.

b) Deuxièmement, on mesurera le spectre sur un tore fermé et sur un tore ouvert ( fig. 5). On observe une diminution de la fréquence de coupure de presque cinq fois sur le tore ouvert. Toujours sous l'hypothèse que $n \tau_{0} \ll 1$ et que $\tau_{0}$ soit presque constant dans les deux cas, on conclut que le nombre d'impulsions par avalanche doit être plus petit de cinq fois aussi dans le tore ouvert. Ce résultat comporte de la (7), une réduction de la densité spectrale aux basses fréquences d'analyse de 100 fois à peu près, puisqu'il y a une diminution aussi de l'amplitude du spectre de l'impulsion élémentaire $\varphi(2 \pi f)$ de l'ordre de 20 fois [4].

c) Troisièmement, on prendra en considération l'amplitude du spectre aux fréquences d'analyse de $25 \mathrm{~Hz}$ et de $5000 \mathrm{~Hz}$, pour des fréquences de magnétisation variables ( $f i g .7$ ). Pour expliquer la non-linéarité de la courbe à $25 \mathrm{~Hz}$, il faut abandonner l'hypothèse que $n \tau_{0}$ soit beaucoup plus petit que l'unité. Le nombre $n$ d'impulsions par seconde est proportionnel à la fréquence de magnétisation, mais le coefficient de proportionnalité n'est pas connu. Pour obtenir la valeur de $n$, il suffit d'imposer la coïncidence d'un point seulement des courbes expérimentale et théorique de l'amplitude du spectre à une basse fréquence d'analyse (par exemple $25 \mathrm{~Hz}$ ) en fonction de la fréquence de magnétisation, ce point étant choisi là où les courbes s'éloignent de la linéarité. Cette simple imposition permet de calculer une valeur unique de $n$ qui assure la coïncidence des courbes expérimentale et théorique sur un vaste champ de fréquences de magnétisation, même pour de différentes fréquences d'analyse, comme montré par la figure 7 .

L'accord qu'on voit exister entre théorie et expérience est donc très bon mais pas encore parfait. En effet, la (7) n'a plus de signification pour $n \tau_{0}$ égal à un, et ça arrive déjà pour des fréquences de magnétisation peu inférieures à un $1 / 10 \mathrm{de} \mathrm{Hz}$, tandis que le spectre reste constant pour des fréquences de magnétisation de quelques hertz.

\section{Spectre de puissance aux fréquences élevées. -} La mesure du spectre devient très difficile aux fréquences de magnétisation pour lesquelles les harmoniques dues à la non-ellipticité du cycle d'hystérésis tombent dans la zone des fréquences d'analyse. En effet, on ne peut employer la technique des deux bobines en opposition, justement à cause du fait que les événements sont corrélés [17]. On n'a pratiquement pas de données expérimentales. Quelques prévisions générales peuvent être faites avec référence à un travail de Bunkin [18, 19].

Si l'on suppose de considérer une succession d'impulsions non corrélées et de fixer un intervalle de temps de durée $t$, le nombre d'impulsions qui tombent dans des intervalles divers ayant tous la même durée $t$ fluctuera selon une courbe à cloche de Gauss centrée sur une valeur moyenne, et avec une variance normale.

Toutefois, dans le cas d'impulsions de Barkhausen créées dans un matériau ferromagnétique alternativement aimanté à saturation, le nombre d'impulsions par cycle est presque constant, de sorte que la fluctua- tion du nombre total d'impulsions pendant des intervalles de durée égale ou multiple de la période est beaucoup plus petite que celle normale. La densité spectrale du bruit aux fréquences d'analyse plus basses que la fréquence de magnétisation est donc très réduite.

Sur ces bases, Bunkin a développé des calculs statistiques dont les résultats sont montrés en figure 11 . Le facteur $\beta$ doit être considéré comme facteur multiplicatif de l'expression donnant le spectre de puissance.

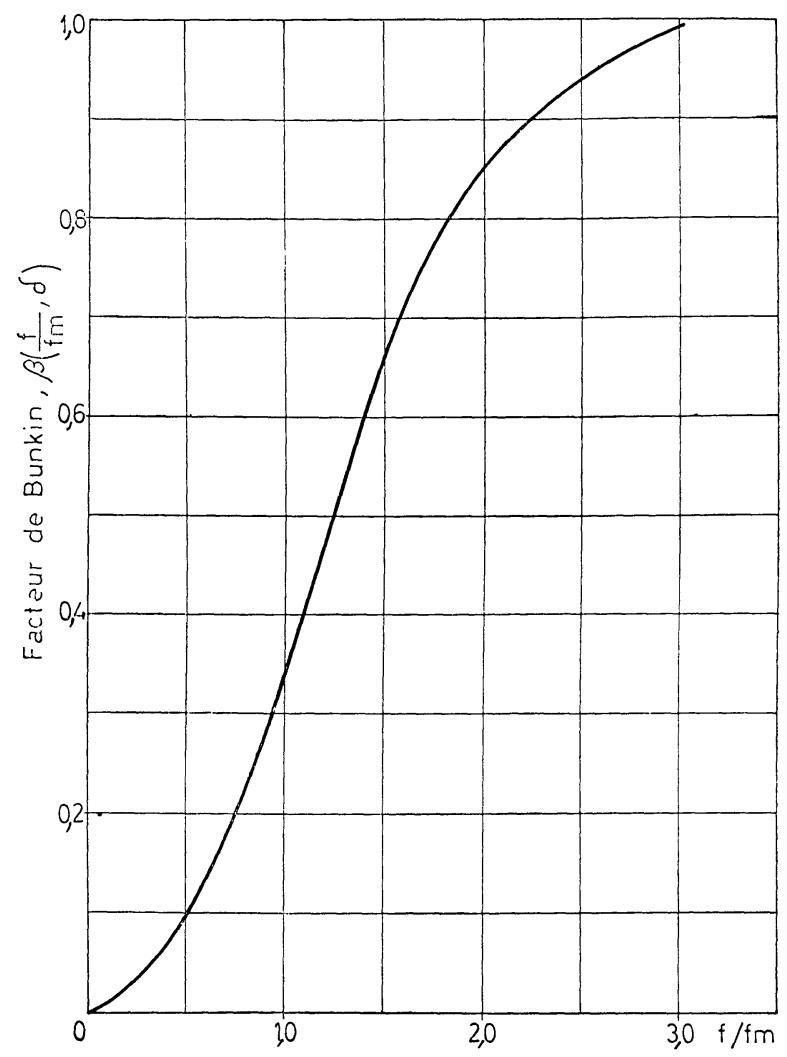

FIG. 11. - Facteur de Bunkin $\beta$ en fonction du rapport entre fréquence d'analyse $f$ et fréquence de magnétisation $f_{\mathrm{m}}$.

5. Conclusions. - La théorie du spectre de puissance du bruit de Barkhausen a été développée de l'expression générale donnée par Mazzetti [11, 12], qui permet d'obtenir le spectre de puissance pour des événements corrélés. On a choisi une fonction de distribution qui représente la tendance des impulsions à se grouper en avalanches. Avec la seule indétermination d'une constante de proportionnalité, on a trouvé un très bon accord entre théorie et expérience aux fréquences de magnétisation inférieures à $1 \mathrm{~Hz}$. Aux fréquences plus élevées, on peut seulement faire des prévisions qualitatives basées sur les résultats de Bunkin.

Les recherches, expérimentales et théoriques, devraient donc continuer. Présentement on ne sait pas donner de critère pour justifier l'amplitude du bruit de Barkhausen dans des matériaux divers, cette amplitude pouvant être très grande ou très petite. On n'est même pas en condition d'établir une relation, même qualitative, entre les valeurs des propriétés magnétiques macroscopiques et l'intensité du bruit. 


\section{BIBLIOGRA PHIE}

[1] StierstadT (K.), Der Magnetische Barkhausen Effekt, Springer Verlag, Berlin, 1966.

[2] Mazzeitit (P.) et Montalentri (G.), J. Appl. Phys., 1963, 34, 3223.

[3] MazzetTi (P.) et MonTalenti (G.), Enevgia Elettrica, 1962, 39, 526.

[4] Mazzeitit (P.) et MontalenNti (G.), Proc. Intern. Conf. on Magnetism, Nottingham, 1964, 701.

[5] Krumhansl (J. A.) et Beyer (R. T.), J. Appl. Phys., 1949, 20, 582 .

[6] Haneman (D.), J. Appl. Phys., 1955, 26, 355.

[7] Biorci (G.) et Pesce't'it (D.), J. Appl. Phys., 1957, 28, 777.

[8] WARREN (K. G.), Electron. Technol., 1961, 38, 89.

[9] Warren (K. G.), Proc. Inst. Elec. Eng., 1964, 111, 387.

[10] VAN DER ZIEL (A.), Fluctuation Phenomena in Semiconductors, Butterworths, London, 1959.
[11] Mazzetuti (P.), Nuovo Cimento, 1962, 25, 1322.

[12] MazzetTi (P.), Nuovo Cimento, 1964, 31, 88.

[13] Sawada (H.), J. Phys. Soc. Japan, 1952, 7, 575.

[14] Bittei (H.) et Wfisterboer (I.), Ann. Phys. (Leipzig), 1959, 7, 203.

[15] Tebble (R. S.) et Newhouse (V. L.), Proc. Phys. Soc., 1953, 66 B, 633.

[16] Bozorth (R. M.), Ferromagnetism, Van Nostrand, New York, 1951, 524.

[17] STORM (L.), Heiden (C.) et Grosse-Nobis (W.), I.E.E.E. Trans. on Magnetics, 1966, MAG-3, 434.

[18] Bunkin (F. W.), Zh. Tekhn. Fiz., 1956, 26, 1791 ; trad. anglaise : Soviet Phys. Techn. Phys., 1956, 1, 1735.

[19] Bunkin (F. W.), Zh. Tekhn. Fiz., 1956, 26, 1782 ; trad. anglaise : Soviet Phys. Techn. Phys., 1956, 1, 1727. 